\title{
Focused ion beam deposited carbon-platinum nanowires for cryogenic resistive thermometry
}

\author{
Kirsten Blagg ${ }^{1}$, Portia Allen ${ }^{1}$, Tzu-Ming Lu ${ }^{2}$, Michael Lilly ${ }^{2}$ and Meenakshi Singh ${ }^{1}$ \\ ${ }^{1}$ Colorado School of Mines, United States, ${ }^{2}$ Sandia National Labs, United States
}

Measuring thermal effects at cryogenic temperatures is the gateway to a large number of relatively uncharted domains such as quantum thermodynamics, quantum thermoelectric effects, quantized heat flows, the thermal Josephson effect, and quantum heat engines. These studies often require the use of nanoscale thermometers with high sensitivity at cryogenic temperatures which can be patterned directly on a device. Historically, a variety of techniques have been used for this including measurements of noise, tunneling current, resistance, and capacitance [1]. Of these techniques, resistive thermometry is particularly popular due to accessible fabrication and the ease of low noise measurements. However, nanoscale, on-chip fabrication of common resistive thermometers requires the use of electron beam lithography (EBL) and stoichiometric deposition. Here, we examine the use of focused ion beam deposited carbon-platinum (FIB C-Pt) composite as a sensitive, local, microthermometer at cryogenic temperatures which can be deposited without the use of a mask at any stage of device fabrication.

FIB deposition is a commonly used to obtain nanoscale, ohmic contacts and has been studied extensively in this role [2]. However, the potential of FIB C-Pt as a cryogenic, resistive thermometer has never been studied. FIB utilizes a simple, high-resolution deposition method to form a granular composite material of platinum inclusions embedded in an amorphous carbon matrix (figure 1a). The C-Pt composite leads to complex conduction characteristics at low temperatures that are independent of either carbon or platinum making them viable for applications as cryogenic resistive thermometers.

FIB C-Pt thermometers were deposited on an RCA cleaned Si substrate with a thermally grown $240 \mathrm{~nm}$ $\mathrm{SiO}_{2}$ layer using a FEI Helios Nanolab. The C-Pt precursor gas, $\left(\mathrm{CH}_{3}\right)_{3}\left(\mathrm{CH}_{3} \mathrm{C}_{5} \mathrm{H}_{4}\right) \mathrm{Pt}$ was heated to $45-$ $46{ }^{\circ} \mathrm{C}$. Each wire was deposited via a $290-300 \mathrm{pC} / \mathrm{mm}$ dose, $30 \mathrm{kV}$ ion voltage, $200 \mathrm{~ns}$ dwell time, $150 \%$ overlap, and a current between $6.5 \mathrm{pA}$ and $226.5 \mathrm{pA}$. Since deposition parameters affect the thermometers' composition and morphology, the FIB C-Pt wires were characterized using transmission electron microscopy (TEM) and energy dispersive x-ray spectroscopy (EDS). The chemical composition of the thermometer was measured as a function of ion beam current (figure 2b). For each deposition, the wire was primarily carbon. As deposition current increases, the carbon content decreases and the amount of platinum increases. Each wire consists of 1.5 - $4 \mathrm{~nm}$ Pt grains embedded in an amorphous carbon matrix (figure 1a). The Pt grain size was found to increase as a function of ion beam current. These differences in composition and structure were found to lead to large changes in conduction and transport mechanisms at room and cryogenic temperatures.

Low temperature resistivity measurements were preformed from $10 \mathrm{mK}$ to $10 \mathrm{~K}$ in a 0 to $8 \mathrm{~T}$ magnetic field using a helium 3 or dilution refrigerator. The resistivity as a function of temperature shows evidence of multiple conduction mechanisms commonly found in metallic granular structures (figure 2a) [3]. In the highest temperature region $(8-10 \mathrm{~K})$, wires deposited at all currents act as highly resistive metals and are poor thermometers. However, as the temperature decreases, the resistance decreases rapidly with temperature for all, but the most metallic samples (high deposition currents). The $24 \mathrm{pA}$ thermometers fall in the idea deposition current range where they are neither metallic like the $44 \mathrm{pA}$ samples, not unpredictable like the $8 \mathrm{pA}$ samples. C-Pt composite wires deposited at $24 \mathrm{pA}$ are excellent thermometers 
in the $10 \mathrm{mK}-1.2 \mathrm{~K}$ range with on average high sensitivity and small magnetic field dependence $(<3 \%)$ comparable to the most widely used cryogenic thermometers (figure $2 \mathrm{~b}$ ). Their performance also remains competitive in the $1.2 \mathrm{~K}-8 \mathrm{~K}$ range. In addition to this wide range of sensitivity and stability, they have the unique advantage of being able to be placed on a chip with $10 \mathrm{~s}$ of $\mathrm{nm}$ precision in a mask less process. Since FIB deposited nanostructures are currently used as electrical probes and can also be used as on-chip joule heaters, these thermometers are amenable for integration with complex devices. The range of unique properties make this $\mathrm{C}$-Pt composite a versatile thermometer for use in myriad classical and quantum thermal measurements.

Acknowledgments: Facility operated for the U.S. Department of Energy (DOE) Office of Science by Los Alamos National Laboratory (Contract DE-AC52-06NA25396) and Sandia National Laboratories (Contract DE-NA-0003525). Sandia National Laboratories is a multimission laboratory managed and operated by National Technology Engineering Solutions of Sandia, LLC, a wholly owned subsidiary of Honeywell International Inc., for the U.S. Department of Energy's National Nuclear Security Administration under contract DE-NA0003525. This work is supported by NSF grant DMR 1807583 and Colorado School of Mines start-up funding.
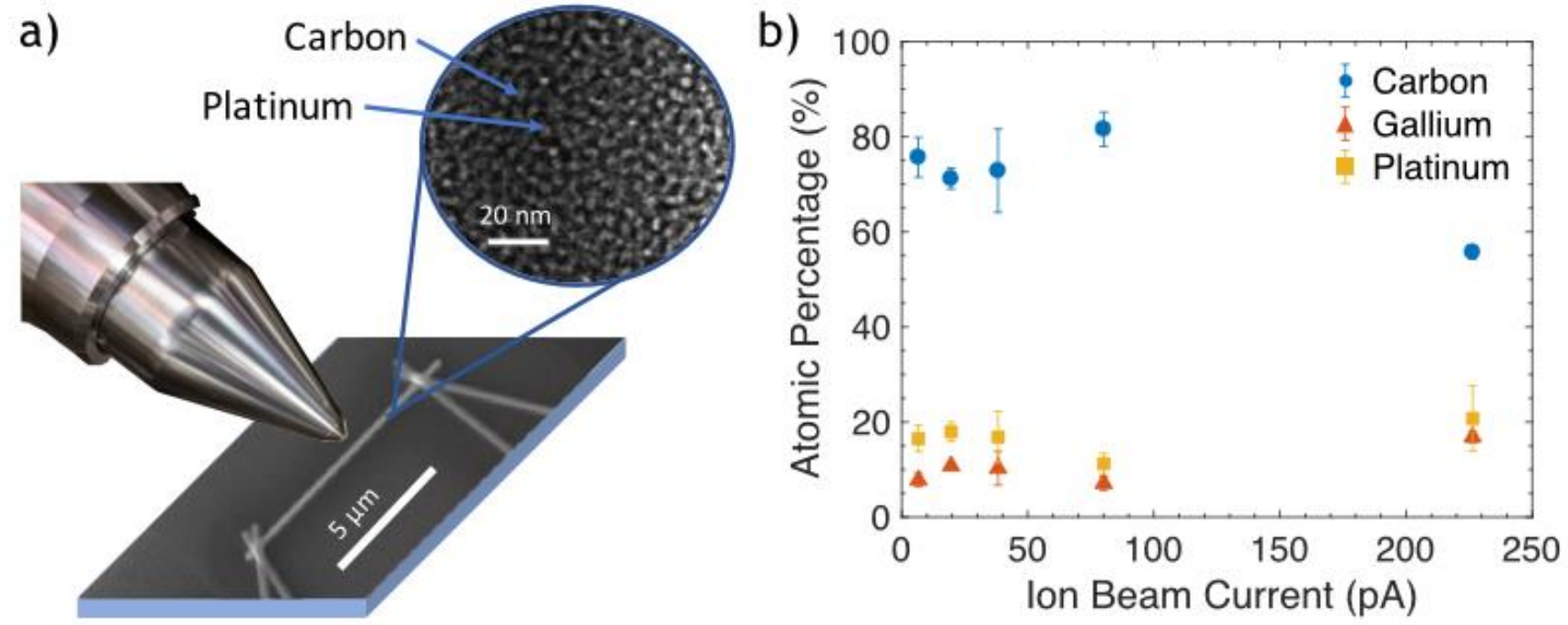

Figure 1. a) Diagram of FIB deposition of a C-Pt nanowire (SEM image). The TEM image of a nanowire deposited at $24 \mathrm{pA}$ as measured via TEM is shown in the circle. The dark spots indicate the granular Pt embedded in a carbon matrix (light background) b) Composition of the FIB deposited wires is shown as cumulative atomic percentage from the EDS spectra of three wires at each deposition current. The data is normalized to exclude the substrate ( $\mathrm{Si}$ and $\mathrm{O}$ ). Carbon is indicated by blue circles, platinum by yellow squares, and gallium by red triangles. 

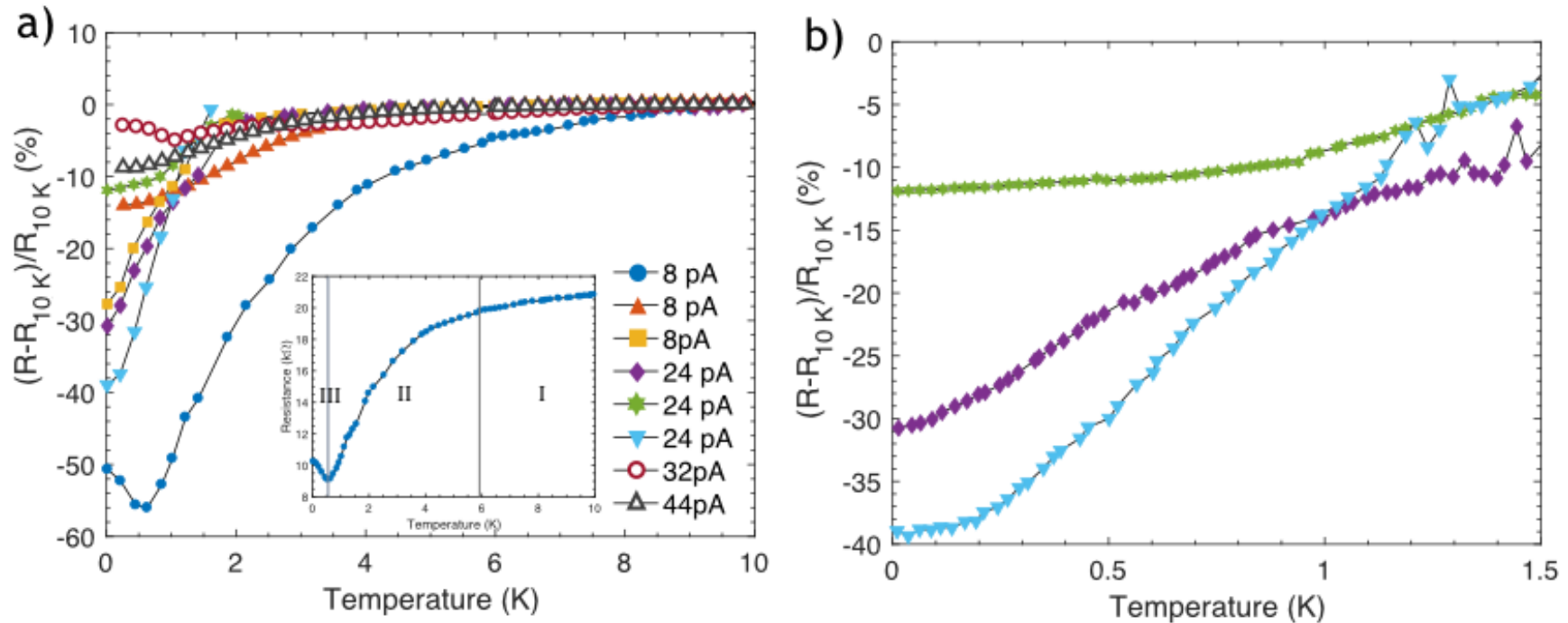

Figure 2. a) Percent change in resistance (R-R10 K/R10 K) of C-Pt deposited at different ion currents measured as a function of temperature. The resistance at $10 \mathrm{~K}$ ranges in value from 0.2 to $14 \mathrm{k} \Omega$. The resistivity characterizations vary between deposition parameters and sample. Inset: Resistance of a FIB $\mathrm{C}$-Pt thermometer deposited at $8 \mathrm{pA}$ as a function of temperature. Conduction is divided into three regions with distinct conduction mechanisms. The boundary between regions is indicative of a qualitative change in transport behavior and is not meant to represent a sharp transition. b) Ultra low temperature resistance of three FIB C-Pt thermometers deposited at 24 pA measured as a function of temperature.

\section{References}

[1] F. Pobell, Matter and methods at low temperatures, Vol. 2, Springer, 2007

[2] A. Fernandez-Pacheco, J. De Teresa, R. Cordoba, M. Ibarra, Metal-insulator transition in pt-c nanowires grown by focused-ion-beam-induced deposition, Physical Review B 79 (17) 174204 (2009).

[3] I. Beloborodov, A. Lopatin, V. Vinokur, K. Efetov, Granular electronic systems, Reviews of Modern Physics 79 (2) 469 (2007) 